\title{
Age-dependent seroprevalence of SARS-CoV-2 antibodies in school-aged children from areas with low and high community transmission
}

Lise Boey ( $\square$ lise.boey@kuleuven.be )

KU Leuven: Katholieke Universiteit Leuven https://orcid.org/0000-0001-9623-4482

\section{Mathieu Roelants}

KU Leuven: Katholieke Universiteit Leuven

Joanna Merckx

McGill University

Niel Hens

Hasselt University: Universiteit Hasselt

Isabelle Desombere

Sciensano

Els Duysburgh

Sciensano

Corinne Vandermeulen

KU Leuven: Katholieke Universiteit Leuven

\section{Research Article}

Keywords: COVID-19, SARS-CoV-2, serosurvey, seroprevalence, schoolchildren, adolescents

Posted Date: March 1st, 2021

DOI: https://doi.org/10.21203/rs.3.rs-221830/v1

License: (1) This work is licensed under a Creative Commons Attribution 4.0 International License. Read Full License

Version of Record: A version of this preprint was published at European Journal of Pediatrics on August 28th, 2021. See the published version at https://doi.org/10.1007/s00431-021-04222-9. 


\section{Abstract}

\section{Background}

It is not yet clear to what extent SARS-CoV-2 infection rates in children reflect community transmission, nor whether infection rates differ between primary schoolchildren and young teenagers.

\section{Methods}

A cross-sectional serosurvey compared the SARS-CoV2 attack-rate in a sample of 362 children recruited from September 21 to October 6, 2020 in primary (ages 6-12) or lower secondary school (ages 12-15) in a municipality with low community transmission (Pelt) to a municipality with high community transmission (Alken) in Belgium. Children were equally distributed over grades and regions. Blood samples were tested for the presence of antibodies to SARS-CoV-2 with an enzyme-linked immunosorbent assay.

\section{Results}

We found anti-SARS-CoV- 2 antibodies in $4.4 \%$ of children in the low transmission region and in $14.4 \%$ of children in the high transmission region. None of the primary schoolchildren were seropositive in the low transmission region, whereas the seroprevalence among primary and secondary schoolchildren did not differ significantly in the high transmission region. None of the seropositive children suffered from severe disease. Children who were in contact with a confirmed case (RR: $3.8 ; 95 \% \mathrm{Cl}: 1.7-8.3$ ), who participated in extracurricular activities (RR: $5.6 ; 95 \% \mathrm{Cl}: 1.2-25.3$ ) or whose caregiver is a healthcare worker who had contact with COVID-19 patients (RR: $2.2 ; 95 \% \mathrm{Cl}: 1.0-4.6$ ), were at higher risk of seropositivity.

\section{Conclusion}

If SARS-CoV2 circulation in the community is high, this will be reflected in the pediatric population with similar infection rates in children aged 6-12 years and 12-15 years.

\section{Introduction}

Seroprevalence studies in children are scarce but the available data indicate that children are less frequently infected compared to adults. Population based studies in Spain and the United States in April and May 2020 reported seroprevalence rates of 0.7 to $5.8 \%$ in children up to 19 years of age, which were generally lower than the rates of 0.7 to $9.9 \%$ observed in adults $[1,2]$. When comparing their results to those of adults, a Swiss study found that the seroprevalence was significantly lower in $5-9$ year old children $(0.8 \%)$ but not significantly different in 10 to 19 years old adolescents (9.6\%) [3]. This indicates that the risk of infection is lower in younger children, but it is not yet elucidated which age marks the transition to infection rates that mimic those in adults, and particularly if children between the age of 12 and 15 years are differently affected compared to those between 6 and 12 years.

In Belgium, the first case of COVID-19 was reported on February $1^{\text {st }}, 2020$. The incidence increased sharply in March, probably driven by people returning from winter holidays. On March $18^{\text {th }}$ the country went in 
lockdown and schools closed. On May, $15^{\text {th }}$ schools partially reopened, which implied that children from the first two years of primary school (ages 6 to 8 years) could attend school four days per week and children from the sixth grade of primary school (ages 11 to 12 years) and last grades of secondary school (ages 1719 years) could attend one or two days per week. School attendance was subject to the following sanitary conditions: physical distancing, hand hygiene, classes of maximum of 10 pupils, and in secondary school also the wear of a face mask. This approach was maintained until the start of the summer holidays (July and August). Schools reopened for all grades on September first with the same sanitary conditions.

From the start, the SARS-CoV-2 pandemic has been characterized by large regional differences in incidence between countries, states, counties and even municipalities. Such large differences occurred even in a small densely populated country like Belgium (Figure 1). However, what is not yet known, is to what extent these infection rates reflect local transmission and how this impacts the prevalence of SARS-CoV- 2 infection in children.

Data on infection in children are important to guide policymakers when deciding which measures to implement to reduce transmission with minimal harm for society. Therefore, we assessed the prevalence of SARS-CoV-2 antibodies in a representative sample of children from primary school and the first three grades of secondary school in a municipality with high and a municipality with low community transmission. In addition, we investigated possible risk factors associated with having SARS-CoV-2 antibodies in this population.

\section{Materials And Methods}

\section{Study setting and population}

Data for this cross-sectional serosurvey were collected in primary (6 - 12 years) and lower secondary (12 15 years) schoolchildren in two Belgian municipalities between September 21 and October 6, 2020. Both municipalities are semi-rural, one (Alken, population 11,500) was among those with the highest SARS-CoV-2 infection rates in Belgium during the first epidemic wave in spring 2020, whereas the other (Pelt, population 32,500 ) had an infection rate below the national average (Figure 1).

\section{Study design and sample size}

A sample of 360 children, evenly divided over both municipalities and primary and secondary schools was planned in order to detect a $6-8 \%$ difference in the prevalence of anti-SARS-CoV-2 antibodies between age groups or municipalities for a baseline prevalence of $5-8 \%$ with a power of $80 \%$. Children were recruited in two primary schools and one secondary school in Pelt, and three primary schools in Alken. Since there is no secondary school in Alken, pupils residing in Alken were recruited in four secondary schools in neighboring municipalities (Hasselt, Stevoort and Herk-de-Stad). Children and their caregivers were invited to the study through a letter that was distributed by the participating schools. Effective participants were randomly chosen among candidates but constrained to an equal distribution by age and sex in each municipality and type of school. Signed informed consent was obtained from caregivers and an informed assent from the 
participating children. A blood sample was taken by venipuncture. Data on socio-demographic characteristics, health status and risk factors for infection were collected with an online questionnaire using LimeSurvey version 2.22.24. Caregivers were invited to complete the questionnaire on the day of sample collection. About three weeks after the initial data collection, an additional questionnaire was sent to obtain information about the profession of caregivers, type of extracurricular activities and travel abroad by family members. The study was approved by the Ethics committee Research UZ/KU Leuven of Leuven, Belgium (s64415).

\section{Laboratory methods}

The blood samples were dispatched to the lab within 24 hours after collection. Following centrifugation, serum aliquots were stored at $-20^{\circ} \mathrm{C}$ until serological analysis. Antibodies directed to the receptor binding domain (RBD) of the structural protein of SARS-CoV-2 were measured using a commercial semi-quantitative enzyme-linked immunosorbent assay (ELISA) (Wantai SARS-CoV-2 Ab ELISA; cat $n^{\circ}$ WS-1096; Beijing Wantai Biological Pharmacy Enterprice Co. Ltd., China), measuring anti-RBD IgG, IgA and IgM concomitantly. As specified by the manufacturer, a ratio is calculated between the optical density (OD) of the patient sample compared to the OD of an internal calibrator. Samples were considered positive at a ratio $\geq 1.1$, the most stringent manufacturer-defined cut-off. Based on in house and reported serum-validations, the estimated specificity and sensitivity, measured $>14$ days post onset of symptoms, for the Wantai ELISA are $99.6 \%(772 / 775 ; 95 \% \mathrm{Cl} 98.9-99.9)$ and $100 \%(155 / 155 ; 95 \% \mathrm{Cl} 97.6-100)$, respectively [4-7]. Laboratory technicians were blinded for information from the questionnaire.

\section{Statistical analysis}

Sample characteristics were compared between municipalities and type of school (primary, secondary) with a t-test for continuous variables, and a chi-squared test or Fisher exact test for categorical variables. Robust standard errors and confidence intervals of prevalence estimates and risks were estimated with generalized estimating equations (GEE) with a binomial distribution and exchangeable correlation structure (compound symmetry) to account for clustering of children in schools and classes. Given the high-performance characteristics of the assay, estimates were not corrected for sensitivity or specificity. Clusters were defined by the class of the current schoolyear. An identity link function was used for the prevalence and a log link function for relative risks (RR). Log transformed estimates were backtransformed (exponentiated) for reporting. Models for the analysis of risk factors were additionally adjusted for municipality (Alken, Pelt) and school level (primary, secondary). A p-value of $5 \%$ or less was considered statistically significant. All data were analyzed with R version 4.0 (R Foundation for Statistical Computing, Vienna, Austria, 2020).

\section{Results}

\section{Study population characteristics}


In total 1,466 children and their caregivers were invited to the study of whom 632 (43.1\%) volunteered, 376 were enrolled, and 362 had a blood sample taken. The online questionnaire was completed by 341 (94.2\%) caregivers and the additional questionnaire by 257 (71.0\%) caregivers. The sample included 175 (48.3\%) boys and $187(51.7 \%$ ) girls (table 1$)$. The mean age at the time of sampling was 10.9 years (range 5.7 14.7 years), with a comparable distribution according to sex and municipality. The number of children per grade in primary school (six grades in total) ranged from 27 to 32 (13 to 16 boys; 12 to 18 girls). In secondary school, 80 (43 boys), 76 (32 boys) and 25 (11 boys) were enrolled in the first, second and third grade, respectively. The third grade of secondary school was only sampled in Alken. In total, $5.8 \%$ of children had a chronic condition, $14.1 \%$ had a family member with a condition that increases the risk of severe COVID-19 infection (e.g. high blood pressure, diabetes...), and 3\% lived in a household with a person at or above 65 years of age. A PCR-confirmed infection was reported for 4 children in the survey (all in March 2020), while parents of 294 participants reported no known history of infection and parents of 43 children reported not knowing whether their child had been infected or not.

Socio-economic characteristics and potential risk factors for SARS-CoV-2 infection are listed in Table 1. These did not differ between the regions or between primary and secondary school, except for use of public transport and summer camps. While rare $(<10 \%)$ in Pelt, almost $30 \%$ of school children in Alken use public transport at least once per week $(p<0.001)$. Public transport is almost exclusively used by secondary schoolchildren ( $n=55 / 165,33.30 \%)$, versus one out of $176(0.6 \%)$ primary schoolchildren. Overnight camps were reported more frequently in secondary school $(98 / 165,59.4 \%)$ compared to primary school $(53 / 176$, $30.1 \%)$. In contrast, day camps were reported more frequently in primary school (53/176, $47.7 \%)$ compared to secondary school $(9 / 165,5.5 \%)$. In total $290(85.0 \%)$ children participated at least one hour per week in extracurricular activities, with an equal distribution by region (Table1) and in primary $(148 / 176,84.1 \%)$ and secondary $(142 / 165,86.1 \%)$ school. About $17.6 \%$ of these activities implied an increased risk (no mask and no distance), and 129 (52.7\%) children participated in indoor activities.

\section{Seroprevalence}

The prevalence of SARS-CoV-2 antibodies by municipality and type of school is listed in Table 2. None of the primary schoolchildren in Pelt were seropositive, however, 8.9\% (8/90) had SARS-CoV-2 antibodies in secondary school. In Alken, we found 13.3\% (12/90) seropositive children in primary and 15.4\% (14/91) seropositive young-adolescents in secondary schools. The overall adjusted RR of having SARS-CoV-2 antibodies was 3.2 (95\%Cl: $1.3-7.9 ; p=0.01)$ in Alken compared to Pelt and $1.6(95 \% \mathrm{Cl}: 0.7-3.8 ; \mathrm{p}=0.3)$ in secondary schools compared to primary schools. The RR of seropositivity in secondary schoolchildren in Alken versus Pelt was 1.7 (95\% Cl: $0.7-4.1 ; p=0.2$ ), and the RR in secondary versus primary school in Alken was 1.1 (95\%Cl: $0.4-1.6 ; p=0.9)$. Comparisons with primary schoolchildren in Pelt are not estimable due the absence of cases. No significant difference was observed in boys versus girls (RR $0.8 ; 95 \% \mathrm{Cl} 0.4-1.5$ ). All but two seropositive children had antibody titers above the upper limit of quantification. All four participants for whom a PCR-confirmed infection was reported in the survey were found seropositive. Among the 294 participants for whom no confirmed infection was reported, 22 (7.5\%) were seropositive, as well as $7(16.3 \%)$ among the 43 whose parents were not sure if their child had been infected. In total, for 
$60.1 \%$ of all pupils, at least one of the following symptoms were reported from February 2020 until data sampling: fever, cough, difficulty breathing, loss of taste, loss of smell, headache, runny nose, sore throat, ear pain, myalgia, asthenia, bellyache, diarrhea, nausea, rash, hyperemia or painful eyes. Importantly, none of the seropositive participants experienced a severe disease requiring hospitalization.

\section{Risk factors for seropositivity}

Table 3 gives an overview of risk factors for SARS-CoV2 seropositivity. We found that children whose caregivers are healthcare workers who were in contact with covid-19 patients are more likely to be seropositive (RR: $2.2 ; 95 \% \mathrm{Cl}: 1.0-4.6)$. Also, children who have at least one hour per week of extracurricular activities are more likely to be seropositive (RR: 5.6; $95 \% \mathrm{Cl}: 1.2-25.3$ ). Based on the second survey we could not establish an association between activities with a high (indoor, without masks, no physical distance) versus low risk (data not shown). Seroprevalence was also higher in children who had contact with a confirmed case (RR: $3.8 ; 95 \% \mathrm{Cl}: 1.7-8.3$ ), had a high-risk contact with a confirmed case (RR:3.7; $95 \% \mathrm{Cl}: 2.0-6.7$ ) or had a high-risk contact with a confirmed case within the household (RR: $5.1 ; 95 \% \mathrm{Cl}: 2.9$ - 9.2). The presence and number of young children, adolescents, adults and elderly persons in the family were not statistically significant associated with the prevalence (risk) of having SARS-CoV-2 antibodies in this cohort.

Even though the study was not designed to investigate clusters, we found an intraclass correlation of 0.15 (SE 0.1). There were 118 classes or clusters in total (mean size is 3.1, range $1-14$ ), but the 91 secondary school students were distributed over 55 clusters (mean cluster size 1.6, range 1 - 4) In total, we found four classes with more than one case. Respectively, two out of four, three out of four, four out of seven and three out of eight children of these classes were seropositive. Assuming an overall prevalence of $9.4 \%$, the binomial probability of observing these numbers varies between $3 / 1000$ and $1 / 10000$.

\section{Discussion}

We found SARS-CoV-2-specific antibodies in $4.4 \%$ of children in a municipality with a low community transmission (Pelt) and in $14.4 \%$ of children living in a municipality with a high community transmission (Alken). The difference in seroprevalence was mainly due to the absence of cases in primary schoolchildren in Pelt where the community transmission preceding the study was low. These findings illustrate that community transmission is reflected in children, and that young children are susceptible when circulation of the virus in the community is high.

Blood samples in our study were collected between September $21^{\text {st }}$ and October $6^{\text {th }} 2020$, which is just before the onset of the second wave in Belgium. Given all four reported PCR-confirmed infections occurred in March 2020 and at that time no control measures were yet in place, we assume that most infections captured by our serosurvey took place during the first wave of the pandemic in Belgium, and most likely prior to the first lockdown which included the closure of schools from March 18, 2020 (Figure 1). 
The overall seroprevalence among children in our study was higher than expected compared to international reports and compared to seroprevalence studies in health care workers and blood donors in Belgium, including samples collected in the same region [1-3, 8-12]. For example, 4.5\% (95\% Cl: $0.2 \%-21.5 \%)$ seropositivity was found in residual samples of 0 - to 10 -year olds and $7.2 \%(95 \% \mathrm{Cl} 1.1 \%-21.6 \%)$ in 10 - to 20-year olds in the province Limburg [10]. However, substandard representativeness and selection bias are a major concern in studies that are not population based or based on a convenience sample. Healthcare workers may be more aware about possible risks and risk avoidance by taking appropriate measures to protect themselves against infection while blood donors are usually more health conscious and may have adopted a more cautious lifestyle in the early days of the pandemic. Both studies may therefore underestimate the true circulation of the SARS-CoV-2 virus in the population.

Our results also indicate a clear difference in seroprevalence between the two studied regions, particularly in primary schoolchildren. Similar regional differences in seroprevalence in children within a geographically small region have been shown in the German state Bavaria [13]. Another unexpected finding was the absence of cases in primary schoolchildren in the low transmission region, while the seroprevalence in this age-group approached the levels observed in older children in the high transmission region. Our data hence suggest that younger and older children are equally susceptible when viral circulation is high but that younger children are less susceptible when circulation is low. A recent study in England showed that there was a strong relation between regional incidence and outbreaks in educational settings, including early years settings ( $<5$-year-olds), primary schools (5-11-year-olds) and secondary schools (11-18-year-olds), with more cases linked to outbreaks in staff than in pupils [14]. All of this emphasizes the importance of mitigation strategies to control community circulation and thus reduce case in schools. This is also supported by an Australian study in care and education centers for children below the age of 18 year, which reported a secondary attack rate of only $1.2 \%$ when confirmed-cases (staff and children) and their contacts from 24 hours before symptom onset were quarantined for 14 days [15]. This study also highlighted the importance of surveillance and contact trancing, testing and quarantining of close contacts within the school setting [15].

Even though our study was not designed to study transmission in schools, the clustering of one third of seropositive cases in only four classes suggests either a common source of infection (e.g. superspreading event), transmission between children, or infection by a staff member before control measures were implemented. Because of this clustering we used robust methods to estimate standard errors of the seroprevalence and for the analysis of possible determinants.

We also found that the risk of having a positive antibody test was almost four times higher in children who were in contact with a confirmed case, especially if this person was an adult household member. Other studies confirm these associations [16-18]. Regarding the higher SARS-CoV-2 seroprevalence in children from healthcare workers who were in contact with COVID-19 patients, we assume that infections took place in March 2020, when there was an overall shortage of personal protective equipment in the health care sector. 
Our study has also limitations. First, a serosurvey gives an overview of past infection but does not allow us to investigate the transmission dynamics within the school environment or onwards transmission in the households. We believe that such studies are much needed to take a final decision regarding measures to curb the spread of SARS-CoV-2, but they require PCR testing and sequencing of active cases and contacts among children, caregivers and school staff during outbreaks in schools. Secondly, we cannot exclude selection-bias due to increased willingness to participate when a family member or close acquaintance was infected. Given that $12 \%$ of parents indicated this to be the case, and another $25 \%$ were not sure, suggests that such risk is real. However, while lower compared to our estimate of the overall seroprevalence, we still found $5.6 \%$ seropositive cases among children for whom the parents did not report any suspicion of a contact.

We conclude that the prevalence of anti-SARS-CoV-2 antibodies in children was higher than expected, that it reflects the virus circulation in the community and that children between the age of 6 and 12 years are equally affected as those between the age of 12 and 15 if community circulation is high.

\section{Abbreviations}

COVID-19 Coronavirus disease 2019

ELISA Enzyme-linked immunosorbent assay

GEE Generalized estimating equations

RBD Receptor binding domain

RR Relative risks

SARS-CoV2 Severe acute respiratory syndrome coronavirus 2

\section{Declarations}

Funding: This study was funded by Sciensano (www.sciensano.be)

Conflicts of interest/Competing interests: Joanna Merckx is an employee of bioMérieux. The other authors declare no conflict of interest.

Availability of data and material: Anonymized data underlying this article will be shared on reasonable request to the corresponding author.

Code availability: not applicable

Authors' contributions: LB, MR, JM, ED and CV designed the study. LB, MR and CV implemented the study and collected data in collaboration with the school health service (Vrij Centrum voor Leerlingbegeleiding $(\mathrm{vCLB})$ ) in Limburg, the participating schools, Wit-Geel Kruis Limburg and the Maria Hospital Pelt. ID was responsible for the laboratory analysis. MR and LB analyzed the data. LB, MR, JM, NH, ID, ED and CV 
interpreted the data. LB and MR wrote the first draft of the manuscript. All authors revised the manuscript and approved the final version.

Ethics approval: The study was approved by the Ethics committee Research UZ/KU Leuven of Leuven, Belgium (s64415) and conducted in accordance with the ethical standards as laid down in the 1964 Declaration of Helsinki and its later amendments.

Consent to participate: Signed informed consent was obtained from caregivers and an informed assent from the participating children.

Consent for publication: not applicable

\section{Acknowledgments}

We are indebted to the several staff members of the school health service (Vrij Centrum voor Leerlingbegeleiding (vCLB)) in Limburg, Belgium, for their cooperation in the recruitment of the schools. We particularly mention Dr. Bea Meertens for her relentless commitment. We are also very thankful for the help and contribution of the management and staff of the participating schools, the home care organization WitGeel Kruis Limburg and the Maria Hospital Pelt. We also acknowledge the lab technicians for performing the serological analyses. Of course, our heartfelt thanks also go to all the children and their caregivers who applied in great numbers to take part in this study.

\section{References}

1. Havers FP, Reed C, Lim T et al (2020) Seroprevalence of Antibodies to SARS-CoV-2 in 10 Sites in the United States, March 23-May 12, 2020. JAMA Intern Med. https://doi.org/10.1001/jamainternmed.2020.4130

2. Pollán M, Pérez-Gómez B, Pastor-Barriuso R et al (2020) Prevalence of SARS-CoV-2 in Spain (ENECOVID): a nationwide, population-based seroepidemiological study. Lancet 396:535-544. https://doi.org/10.1016/S0140-6736(20)31483-5

3. Stringhini S, Wisniak A, Piumatti G et al (2020) Seroprevalence of anti-SARS-CoV-2 IgG antibodies in Geneva, Switzerland (SEROCoV-POP): a population-based study. Lancet 396:313-319. https://doi.org/10.1016/S0140-6736(20)31304-0

4. Lassaunière R, Frische A, Harboe Z et al (2020) Evaluation of nine commercial SARS-CoV-2 immunoassays. medRxiv 2020.04.09.20056325. https://doi.org/10.1101/2020.04.09.20056325

5. Lisboa Bastos M, Tavaziva G, Abidi SK et al (2020) Diagnostic accuracy of serological tests for covid19: Systematic review and meta-analysis. BMJ 370:2516. https://doi.org/10.1136/bmj.m2516

6. GeurtsvanKessel CH, Okba NMA, Igloi Z et al (2020) An evaluation of COVID-19 serological assays informs future diagnostics and exposure assessment. Nat Commun 11:. https://doi.org/10.1038/s41467-020-17317-y

7. Zhao J, Yuan Q, Wang H et al (2020) Antibody Responses to SARS-CoV-2 in Patients With Novel Coronavirus Disease 2019. Clin Infect Dis 71:2027-2034. https://doi.org/10.1093/cid/ciaa344 
8. Dingens AS, Crawford KHD, Adler A et al (2020) Serological identification of SARS-CoV-2 infections among children visiting a hospital during the initial Seattle outbreak. Nat Commun 11:. https://doi.org/10.1038/s41467-020-18178-1

9. Posfay-Barbe KM, Andrey DO, Virzi J et al (2020) Prevalence of IgG against SARS-CoV-2 and evaluation of a rapid MEDsan IgG test in children seeking medical care. Clin Infect Dis. https://doi.org/10.1093/cid/ciaa1702

10. Herzog S, De Bie J, Abrams S et al (2020) Seroprevalence of IgG antibodies against SARS coronavirus 2 in Belgium - a serial prospective cross-sectional nationwide study of residual samples. medRxiv 2020.06.08.20125179. https://doi.org/10.1101/2020.06.08.20125179

11. Mortgat L, Barbezange C, Fischer N et al (2020) Title: SARS-CoV-2 Prevalence and Seroprevalence among Healthcare Workers in Belgian Hospitals: Baseline Results of a Prospective Cohort Study Authors*. medRxiv 2020.10.03.20204545. https://doi.org/10.1101/2020.10.03.20204545

12. Sciensano (public health Belgium) Aantal bloeddonoren en gezondheidswerkers met antistoffen tegen coronavirus blijft stabiel | sciensano.be. https://www.sciensano.be/nl/pershoek/aantal-bloeddonorenen-gezondheidswerkers-met-antistoffen-tegen-coronavirus-blijft-stabiel. Accessed 11 Jan 2021

13. Hippich M, Holthaus L, Assfalg R et al (2020) Public health antibody screening indicates a six-fold higher SARS-CoV-2 exposure rate than reported cases in children. Med. https://doi.org/10.1016/j.medj.2020.10.003

14. Ismail SA, Saliba V, Lopez Bernal J et al (2020) SARS-CoV-2 infection and transmission in educational settings: a prospective, cross-sectional analysis of infection clusters and outbreaks in England. Lancet Infect Dis. https://doi.org/10.1016/S1473-3099(20)30882-3

15. Macartney K, Quinn HE, Pillsbury AJ et al (2020) Transmission of SARS-CoV-2 in Australian educational settings: a prospective cohort study. Lancet Child Adolesc Heal 4:807-816. https://doi.org/10.1016/S2352-4642(20)30251-0

16. Gudbjartsson DF, Norddahl GL, Melsted P et al (2020) Humoral Immune Response to SARS-CoV-2 in Iceland. N Engl J Med 383:1724-1734. https://doi.org/10.1056/nejmoa2026116

17. Waterfield T, Watson C, Moore R et al (2020) Seroprevalence of SARS-CoV-2 antibodies in children: A prospective multicentre cohort study. Arch Dis Child 0:1-7. https://doi.org/10.1136/archdischild-2020320558

18. Torres JP, Piñera C, De La Maza V et al (2020) Severe Acute Respiratory Syndrome Coronavirus 2 Antibody Prevalence in Blood in a Large School Community Subject to a Coronavirus Disease 2019 Outbreak: A Cross-sectional Study. Clin Infect Dis. https://doi.org/10.1093/cid/ciaa955

19. Sciensano Epistat - COVID-19 Belgian Dashboard. https://epistat.wiv-isp.be/covid/covid-19.html. Accessed 12 Jan 2021

\section{Tables}

Table 1: Participant characteristics and risk factors for SARS-CoV2 infection (questionnaire) 


\begin{tabular}{|c|c|c|c|}
\hline & $\begin{array}{l}\text { Alken } \\
(\mathrm{n}=172)\end{array}$ & $\begin{array}{l}\text { Pelt } \\
(n=169)\end{array}$ & $\begin{array}{l}\text { Total } \\
(\mathrm{n}=341)\end{array}$ \\
\hline Personal data & $\mathrm{n}(\%)$ & $\mathrm{n}(\%)$ & n (\%) \\
\hline Sex: Boys & $86(47.5 \%)$ & $89(49.2 \%)$ & $175(48.3 \%)$ \\
\hline \multicolumn{4}{|l|}{ Socio-economic characteristics } \\
\hline \multicolumn{4}{|l|}{ Family context } \\
\hline Two-parent family & $154(89.5)$ & $237(87.0)$ & 301 (88.3) \\
\hline Other $^{\text {a }}$ & $18(10.5)$ & $12(13.1)$ & $40(11.8)$ \\
\hline Child lives also elsewhere & $21(12.2)$ & $25(14.8)$ & $46(13.5)$ \\
\hline Mother with college degree & $145(84.3)$ & $111(65.7)$ & $256(75.1)$ \\
\hline Father with college degree & $110(64.0)$ & $92(54.5)$ & $202(59.2)$ \\
\hline \multicolumn{4}{|l|}{ Origin } \\
\hline Belgian & 157 (91.3) & $142(84.0)$ & $299(87.7)$ \\
\hline Dutch & $4(2.3)$ & $21(12.4)$ & $36(7.3)$ \\
\hline Other & $11(6.4)$ & $6(3.6)$ & $17(5.0)$ \\
\hline Disposable family income $>3000$ euro / month & $126(73.3)$ & $107(63.3)$ & $233(68.3)$ \\
\hline \multicolumn{4}{|l|}{ Personal and family risk factors } \\
\hline Shared bedroom ${ }^{\mathrm{b}}$ & $16(9.3)$ & $24(14.2)$ & $40(11.7)$ \\
\hline \multicolumn{4}{|l|}{ Public transport use ${ }^{\mathrm{C}}$} \\
\hline Regularly (> 1/week) & $46(26.7)$ & $10(5.9)$ & $46(16.4)$ \\
\hline Occasionally (< 1/week) & $126(73.3)$ & $159(94.1)$ & $285(83.6)$ \\
\hline \multicolumn{4}{|l|}{ Participated in summer camp } \\
\hline Overnight camp & $94(54.7)$ & $88(52.1)$ & $182(53.4)$ \\
\hline Day camp & $28(16.3)$ & $34(20.1)$ & $62(18.2)$ \\
\hline No & $50(29.1)$ & $47(27.8)$ & $97(28.4)$ \\
\hline Extracurricular activities & $153(89.0)$ & $148(87.6)$ & $301(88.3)$ \\
\hline Household member who traveled to a risk region d,e & $33(26.4)$ & $17(14.2)$ & $50(20.5)$ \\
\hline Caregiver is HCW in contact with COVID-19 patients ${ }^{d}$ & $25(19.7)$ & $16(13.3)$ & $41(16.7)$ \\
\hline
\end{tabular}

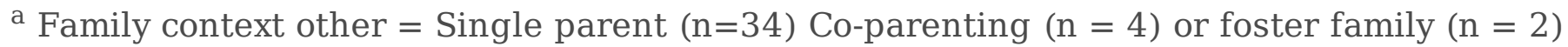

$\mathrm{b}$ In each region there are 2 children who share the room with a person of 15 years or older; All the others share their bedroom with another child $<15$ years.

c Significantly different between Alken and Pelt (chi square test $\mathrm{p}<0.001$ )

$\mathrm{d}$ Based on respondents of additional survey ( $\mathrm{n}=257 ; 127$ in Alken and 120 in Pelt)

e Travel to a high-risk region since February 2020

Table 2: Prevalence of SARS-CoV-2 antibodies by municipality and school (primary versus seconda school)

\begin{tabular}{lccc}
\hline & Alken & Pelt & Both municipalities \\
\hline Primary schools, n pupils & $\mathbf{9 0}$ & $\mathbf{9 1}$ & $\mathbf{1 8 1}$ \\
Seropositive, \% (95\%CI) & $13.3(3.2-23.5)$ & $0(0-4.1)$ & $6.6(1.2-12.1)$ \\
\hline Secondary Schools, n pupils & $\mathbf{9 1}$ & $\mathbf{9 0}$ & $\mathbf{1 8 1}$ \\
Seropositive, \% (95\%CI) * & $15.4(8.6-22.2)$ & $8.9(2.1-15.7)$ & $12.2(7.2-17.1)$ \\
\hline Total, n pupils & $\mathbf{1 8 1}$ & $\mathbf{1 8 1}$ & $\mathbf{3 6 2}$ \\
\hline Seropositive, \% (95\%CI) * & $14.4(8.2-20.5)$ & $4.4(0.7-8.1)$ & $9.4(5.7-13.1)$ \\
\hline
\end{tabular}


Table 3: Risk factors for seropositivity

\begin{tabular}{|c|c|c|}
\hline & Ratio (\%) seropositive & RR $(95 \% \mathrm{CI})^{\mathrm{a}}$ \\
\hline \multicolumn{3}{|l|}{ Public transport } \\
\hline Frequent use ( $>1 /$ week) & 9/56 (16.1) & $1.1(0.5-2.5)$ \\
\hline Occasional use $(<1 /$ week $)$ & $47 / 285(8.4)$ & Reference \\
\hline \multicolumn{3}{|l|}{ Summer camps } \\
\hline Overnight camp & 20/182 (11.0) & $1.7(0.7-3.9)$ \\
\hline Day time camp & $6 / 62(9.7)$ & $2.7(1.0-7.3)$ \\
\hline No camp & $7 / 97(7.2)$ & Reference \\
\hline \multicolumn{3}{|l|}{ Extracurricular activities } \\
\hline$>1$ hour/week & $32 / 290(11.0)$ & $5.6(1.2-25.3)$ \\
\hline$<1$ hour/week & $1 / 51(2.0)$ & Reference \\
\hline \multicolumn{3}{|l|}{ Shared bedroom } \\
\hline No Shared bedroom & $28 / 301(9.3)$ & Reference \\
\hline Shared bedroom & $5 / 40(12.5)$ & $1.7(0.8-3.4)$ \\
\hline \multicolumn{3}{|l|}{ Contact with a confirmed case } \\
\hline Contact with a confirmed case ${ }^{b}$ & $11 / 41(27)$ & $2.9(1.6-5.4)$ \\
\hline Contact with an infected household member ${ }^{\mathrm{c}}$ & $8 / 19(42)$ & $5.1(2.9-9.2)$ \\
\hline High risk contact ${ }^{d}$ & $10 / 29(34.5)$ & $3.7(2.0-6.7)$ \\
\hline \multicolumn{3}{|l|}{ Profession of caregiver ${ }^{e}$} \\
\hline HCW with contact with Covid-19 patients & $9 / 41(22)$ & $2.2(1.0-4.6)$ \\
\hline HCW without contact with Covid-19 patients & $4 / 41(9)$ & $1.2(0.4-3.4)$ \\
\hline Non-HCW & $14 / 159(9)$ & Reference \\
\hline \multicolumn{3}{|l|}{ Travel ${ }^{e}$} \\
\hline Household member who traveled to a risk region ${ }^{f}$ & $7 / 50(14)$ & $1.2(0.5-2.6)$ \\
\hline No household member travelled to a risk region & 20/195 (10.3) & Reference \\
\hline
\end{tabular}

a Adjusted for municipality (Alken versus Pelt) and type of school (primary versus secondary) as fixed covariables; and class as random factor

${ }^{\mathrm{b}}$ Contact with confirmed case versus no known contact

C Contact with an infected household member; RR versus no contact with an infected household member

d High risk: no mask, no social distancing $(<1.5 \mathrm{~m})$, for more than 15 minutes; RR versus no high-risk contact

e Based on respondents of additional survey

$\mathrm{f}$ Travel to a high-risk region since February 2020 
Figures

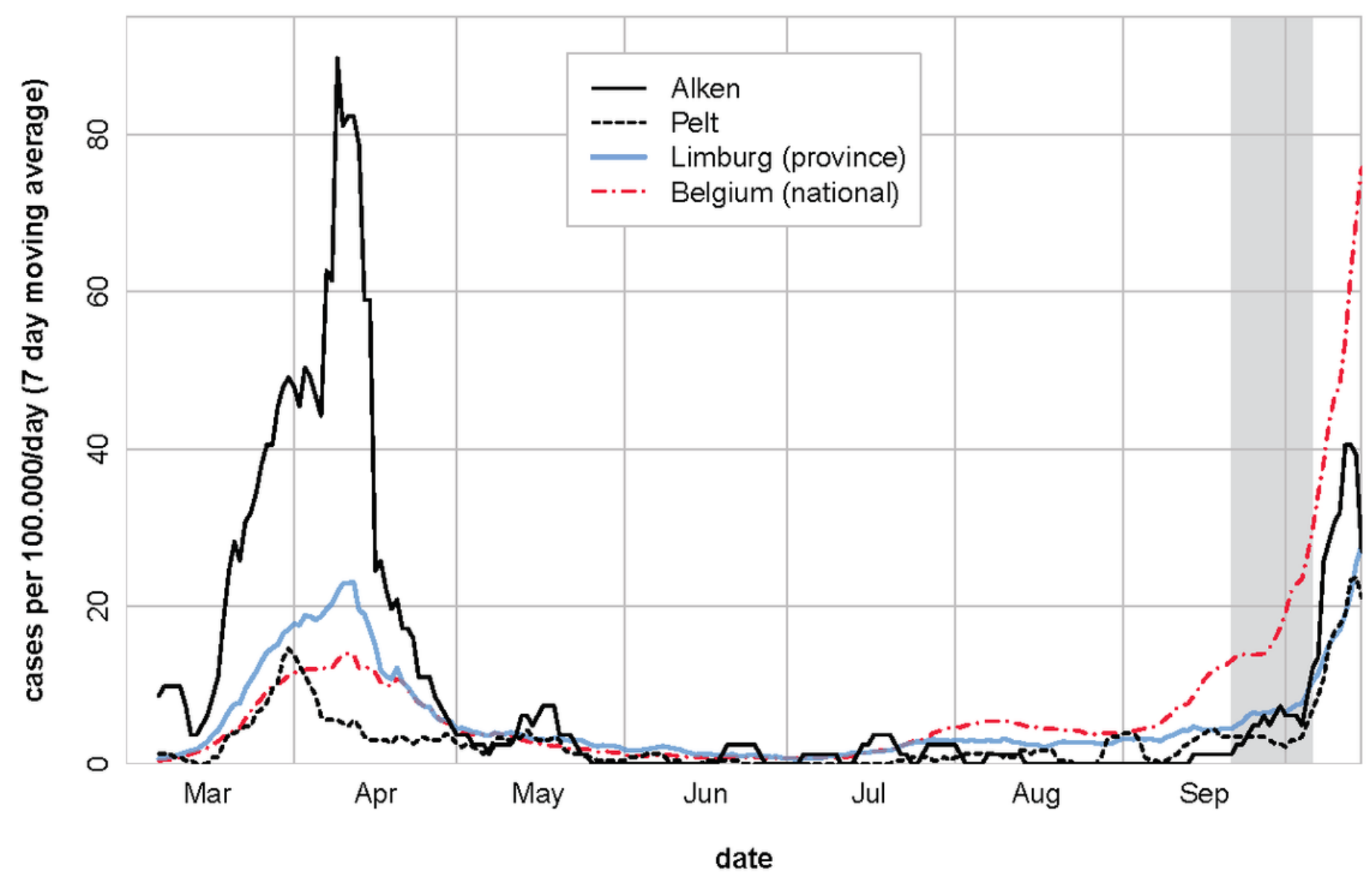

Figure 1

Mean number of confirmed SARS-CoV-2 infections per 100,000 inhabitants during the previous 7 days in Pelt and Alken, the province (Limburg) to which they belong and the whole country (Belgium) from March 1 to October 15, 2020 [19].The grey area represents the study period from September 21 to October 6. 